\title{
NWI open Hypogonadism and low bone mineral density in patients on long-term intrathecal opioid delivery therapy
}

\author{
Rui V Duarte, ${ }^{1,2}$ Jon H Raphael, ${ }^{1,2}$ Jane L Southall, ${ }^{2}$ Mourad H Labib, ${ }^{3}$ \\ Andrew J Whallett, ${ }^{4}$ Robert L Ashford ${ }^{1}$
}

To cite: Duarte RV, Raphael JH, Southall JL, et al. Hypogonadism and low bone mineral density in patients on long-term intrathecal opioid delivery therapy. BMJ Open 2013;3: e002856. doi:10.1136/ bmjopen-2013-002856

- Prepublication history for this paper is available online. To view these files please visit the journal online (http://dx.doi.org/10.1136/ bmjopen-2013-002856).

Received 8 March 2013 Revised 4 May 2013 Accepted 7 May 2013

This final article is available for use under the terms of the Creative Commons Attribution Non-Commercial 2.0 Licence; see http://bmjopen.bmj.com

\footnotetext{
${ }^{1}$ Faculty of Health, Birmingham City University, Birmingham, UK ${ }^{2}$ Department of Pain Management, Russells Hall Hospital, Dudley, UK ${ }^{3}$ Department of Clinical Biochemistry, Russells Hall Hospital, Dudley, UK ${ }^{4}$ Department of Rheumatology, Russells Hall Hospital, Dudley, UK
}

Correspondence to Dr Rui V Duarte; ruivduarte@gmail.com

\section{ABSTRACT}

Objectives: This study aimed to investigate the hypothalamic-pituitary-gonadal axis in a sample of male patients undertaking intrathecal opioid delivery for the management of chronic non-malignant pain and the presence of osteopaenia and/or osteoporosis in those diagnosed with hypogonadism.

Design: Observational study using health data routinely collected for non-research purposes.

Setting: Department of Pain Management, Russells Hall Hospital, Dudley, UK.

Patients: Twenty consecutive male patients attending follow-up clinics for intrathecal opioid therapy had the gonadal axis evaluated by measuring their serum luteinising hormone, follicle stimulating hormone, total testosterone, sex hormone binding globulin and calculating the free testosterone level. Bone mineral density was measured by DEXA scanning in those patients diagnosed with hypogonadism.

Results: Based on the calculated free testosterone concentrations, $17(85 \%)$ patients had biochemical hypogonadism with 15 patients $(75 \%)$ having free testosterone $<180 \mathrm{pmol} / \mathrm{L}$ and 2 patients $(10 \%)$ between 180 and $250 \mathrm{pmol} / \mathrm{L}$. Bone mineral density was assessed in 14 of the 17 patients after the exclusion of 3 patients. Osteoporosis (defined as a T score $\leq-2.5 \mathrm{SD}$ ) was detected in three patients (21.4\%) and osteopaenia (defined as a T score between -1.0 and $-2.5 \mathrm{SD}$ ) was observed in seven patients $(50 \%)$. Five of the 14 patients $(35.7 \%)$ were at or above the intervention threshold for hip fracture.

Conclusions: This study suggests an association between hypogonadism and low bone mass density in patients undertaking intrathecal opioid delivery for the management of chronic non-malignant pain.

Surveillance of hypogonadism and the bone mineral density levels followed by appropriate treatment may be of paramount importance to reduce the risk of osteoporosis development and prevention of fractures in this group of patients.

\section{INTRODUCTION}

Treatment strategies for the management of chronic pain start with the lowest risk and

\section{ARTICLE SUMMARY}

Article focus

- Hypogonadism is common in intrathecal opioid therapy patients, but there is a dearth of literature investigating bone mineral density in this population.

- We aimed to prospectively investigate if undiagnosed hypogonadism in intrathecal opioid therapy patients may result in low bone mineral density levels.

Key messages

- Undiagnosed hypogonadism in intrathecal opioid therapy patients may lead to low bone mineral density levels.

- Hypogonadism and bone mineral density levels surveillance may be of paramount importance to reduce the risk of osteoporosis and fractures in patients undertaking intrathecal opioid therapy.

Strengths and limitations of this study

- To our knowledge, this is the first study to specifically address the potential decrease in bone mineral density as a consequence of long-term intrathecal opioid therapy.

- Further studies are warranted to assess the effectiveness of early detection and adequate treatment to prevent bone mineral density decrease and to investigate the value of hormonal replacement therapy to normalise bone mineral density levels.

least invasive intervention and progress if a treatment is not effective. Intrathecal drug delivery (IDD) is considered a last resort treatment for the management of severe chronic pain owing to its invasive nature, concerns about long-term opioid use and the possible complications related to this modality of treatment. Intrathecal spinal analgesia has become a recognised treatment for chronic non-malignant pain since the first reservoir was implanted in $1981 .^{1}$ The use of opioids via intrathecal drug delivery allows for a selective concentration to reach an 
important site of pain transmission, the spinal cord dorsal horn. ${ }^{2}$ Opioid administration into the intrathecal space achieves its effects at lower doses than by using the epidural route. ${ }^{3}$ The drug is highly localised, so its analgesic efficacy is maximised at lower doses. ${ }^{4}$ Moreover, opioid dose escalation over the years may be modest. ${ }^{5} 6$ These factors lead to a decrease in the possible opioid side effects.

The potential effect of intrathecal opioid delivery on the endocrine system is one that is least recognised and investigated. ${ }^{7}$ Currently, opioid-induced hypogonadism is under-recognised and undertreated. ${ }^{8}{ }^{9}$ Some patients may attribute the signs and symptoms of hypogonadism, such as decreased libido, tiredness, loss of muscle mass and strength, among others, to the chronic pain and its related conditions rather than to the intrathecal medication. ${ }^{710}$ Moreover, symptoms of hypogonadism are often neither disclosed by the patient nor documented by the physician. ${ }^{11}$ Currently, limited clinical awareness of the opioid effects on the endocrine system, together with the lack of information on their long-term consequences, is likely to result in a lack of information provision to the patient when long-term opioid therapy is being considered. ${ }^{12}$

Animal studies suggest that opioids affect gonadotropin release via the inhibition of the gonadotropin releasing hormone $(\mathrm{GnRH})$ by $\beta$-endorphin, ${ }^{13}$ most likely at the $\mu$-receptors. ${ }^{14}$ This inhibition reduces the release of the luteinising hormone (LH) and the follicle stimulating hormone (FSH) by the pituitary gland and consequently the production of testosterone by the gonads, resulting in hypogonadism and loss of libido. This is characterised by the low or low-normal LH and FSH levels and low testosterone levels. ${ }^{11}$ Most of the circulating testosterone is bound to the sex hormone-binding globulin (SHBG). A lesser fraction is non-specifically bound to albumin and only a small percentage of the testosterone is unbound. ${ }^{15}$ Historically, free testosterone was thought to be the only biologically-active component. However, testosterone is weakly bound to serum albumin and dissociates freely in the capillary bed, as well as being readily available for tissue uptake. All non-SHBG-bound testosterone is therefore considered bioavailable.

Hypogonadism is an important risk factor for development of osteoporosis in both sexes, but opioids have not been considered as a risk factor. ${ }^{16}$ To our knowledge, the assessment of bone mineral density (BMD) in patients undertaking intrathecal drug delivery is limited to one study, which suggested a tendency towards decreased BMD in these patients, but the prevalence of osteopaenia or osteoporosis in these patients was not reported in this study. ${ }^{17}$

The aim of this study was to investigate the hypothalamic-pituitary-gonadal axis in a sample of male patients undertaking intrathecal opioid delivery for the management of chronic non-malignant pain and to assess the prevalence of osteopaenia and/or osteoporosis in those diagnosed with hypogonadism.

\section{METHODS}

\section{Patients}

Twenty consecutive male patients attending follow-up clinics for IDD therapy at Russells Hall Hospital, Dudley, $\mathrm{UK}$, for the management of chronic non-cancer pain were included in this observational study using health data routinely collected for non-research purposes. All assessments were performed as part of routine clinical care. No additional procedures were carried out for research purposes. None of these patients received testosterone supplementation within the previous 3 months. The pain syndrome experienced by the patients was classified as nociceptive $(58.8 \%)$, neuropathic $(5.8 \%)$ and mixed nociceptive-neuropathic $(35.3 \%)$. All the patients were receiving intrathecal opioids for the management of their pain. Intrathecal morphine was the only medication administered to $50 \%$ of the sample. In individual cases, other substances were added to the intrathecal medication, with combinations of morphine with bupivacaine $(12.5 \%)$, morphine with bupivacaine and clonidine (25\%), and morphine with bupivacaine and baclofen (12.5\%).

\section{Laboratory methods}

Blood samples were collected between 08:00 and 11:00 during a 7-month period (April-October 2010), as part of routine clinical care, for the measurement of serum LH, FSH, prolactin (PRL), total testosterone (TT) and SHBG. All assays were carried out by the Department of Clinical Biochemistry at Russells Hall Hospital, Dudley, UK. LH, FSH, PRL and TT were measured according to the manufacturer's instructions by immunoenzymometric fluorimetric assay on the Tosoh AIA 2000 LA analyser (Tosoh Bioscience N.V., Tessenderlo, Belgium). The inter-assay imprecision (\%CV) quoted by the manufacturer was $2.6 \%$ for $\mathrm{LH}, 2.3 \%$ for $\mathrm{FSH}$ and $5.3 \%$ for testosterone. SHBG was measured according to the manufacturer's instructions by chemiluminescent immunometric assay on the Immulite $2000 \mathrm{XPi}$ analyser (Siemens Healthcare Diagnostics Ltd, Camberley, Surrey, UK). The inter-assay variability $(\% \mathrm{CV})$ for SHBG was $5 \%$. Calculations of free testosterone (FT) were carried out using the Vermeulen equation. ${ }^{15}$ Serum TT $<8 \mathrm{nmol} / \mathrm{L}$ and/or FT $<180 \mathrm{pmol} / \mathrm{L}$ was considered as biochemical hypogonadism. Serum TT 8-12 nmol/L and/or FT 180$250 \mathrm{pmol} / \mathrm{L}$ were considered as borderline/low. ${ }^{18}$

\section{Assessment of bone mineral density}

Bone mineral density was measured by DEXA scanning of the femur (neck and hip) and lumbar spine or left forearm using the Lunar Prodigy DEXA (GE Lunar Corp., Madison, Wisconsin, USA). Bone densitometry DEXA scans were carried out by the Department of Radiology at Corbett Hospital, Dudley, UK. A lumbar spine scan was not carried out on patients who had previous spinal surgery. In those cases, assessment was performed at the left forearm site. Results are presented as $\operatorname{BMD}\left(\mathrm{g} / \mathrm{cm}^{2}\right), \mathrm{T}$ and $\mathrm{Z}$ scores. Reference values for 
T scores were based on the UK (ages 20-40) femur, spine or forearm reference population (v107). Osteopaenia was defined as a $\mathrm{T}$ score between -1.0 and $-2.5 \mathrm{SD}$, and osteoporosis as a $\mathrm{T}$ score at or below -2.5 SD. Measurements of height, weight and body mass index (BMI) were also performed. The BMI scores were categorised according to the WHO key cut-off points as $<18.5$ (underweight), $\geq 18.5$ and $\leq 24.9$ (normal weight), $\geq 25$ and $\leq 29.9$ (overweight) and $\geq 30 \mathrm{~kg} / \mathrm{m}^{2}$ (obese). The 10-year probability of fracture was calculated based on the Fracture Risk Assessment Tool (FRAX). ${ }^{19}$ In addition to the BMD value or T score (femoral neck), this tool takes into account clinical risk factors for the development of osteoporotic or hip fractures such as previous fractures, a history of hip fracture in the patient's parents and hypogonadism, among other factors.

\section{Data analysis}

A Kolmogorov-Smirnov test was performed to test the distribution of numerical data, followed by the appropriate statistical tests. Comparisons between groups were carried out with the Mann-Whitney test. Data are reported as median (minimum-maximum). The 95\% CIs for the TT and FT median values were calculated for comparison with normal reference values. Statistical significance was judged at the $5 \%$ level. Statistical tests were performed using the Predictive Analytics SoftWare (PASW) (V.18.0, SPSS Inc, Chicago, Illinois, USA).

\section{RESULTS}

\section{Assessment of sex hormones}

The median age at the time of blood collection was 58 years (47-69). The median duration from implantation of the IDD system to hormone assay was 100 months (15-203) with an intrathecal opioid dose of $2.68 \mathrm{mg} /$ day (range 1-9.7) (table 1). The duration of pain prior to the start of IDD was 9 years (range 3-35).

The median TT level with $95 \%$ CIs was $4.95 \mathrm{nmol} / \mathrm{L}$ (3.0 to 10.1), which was significantly lower than the cut-off level of $12 \mathrm{nmol} / \mathrm{L}$ for borderline/low testosterone. The median FT levels with $95 \%$ CIs (69.45 (47.3 to 127.0))

Table 1 Reference ranges and levels in 20 men undertaking intrathecal opioid administration

\begin{tabular}{lll}
\hline & $\begin{array}{l}\text { Reference } \\
\text { range }\end{array}$ & $\begin{array}{l}\text { Intrathecal opioid } \\
\text { patients }\end{array}$ \\
\hline $\mathrm{LH}(\mathrm{IU} / \mathrm{L})$ & $2.2-13.3$ & $1.9(0.2-19.9)$ \\
$\mathrm{FSH}(\mathrm{IU} / \mathrm{L})$ & $1-7$ & $5.3(0.3-23.9)$ \\
$\mathrm{SHBG}(\mathrm{nmol} / \mathrm{L})$ & $13-71$ & $51(17-123)$ \\
$\mathrm{PRL}(\mathrm{mU} / \mathrm{L})$ & $0-445$ & $225(53-614)$ \\
$\mathrm{TT}(\mathrm{nmol} / \mathrm{L})$ & $9.47-28.3$ & $4.95(1.2-18.8)$ \\
$\mathrm{FT}(\mathrm{pmol} / \mathrm{L})$ & $185-437$ & $69.45(14-328)$ \\
\hline
\end{tabular}

Statistics are presented as median (minimum-maximum). FSH, follicle stimulating hormone; FT, free testosterone; LH, luteinising hormone; PRL, prolactin; SHBG, sex hormone binding globulin; TT, total testosterone. were also significantly lower than the cut-off level of $180 \mathrm{pmol} / \mathrm{L}$ for low FT $(\mathrm{t}=-3.403, \mathrm{p}<0.005, \mathrm{r}=0.61)$. The mean LH, FSH and SHBG concentrations were within the respective reference ranges. Prolactin levels were above the reference range in two patients. One of these patients had low TT and FT values and the other patient presented a borderline/low TT value. Based on TT calculations, 17 (85\%) patients presented biochemical hypogonadism values with $12(60 \%)$ at less than $8 \mathrm{nmol} / \mathrm{L}$ and $5(25 \%)$ presented with TT values between 8 and $12 \mathrm{nmol} / \mathrm{L}$ (borderline/low). Based on FT calculations, 17 (85\%) patients were biochemically hypogonadal with $15(75 \%)$ patients at less than $180 \mathrm{pmol} / \mathrm{L}$ and two (10\%) patients between 180 and $250 \mathrm{pmol} / \mathrm{L}$. Only one of the patients had TT and FT values within the quoted reference ranges, two patients presented borderline/low TT and normal FT values, one patient had low FT values and normal TT values, and one patient had borderline/low FT values and normal TT values.

\section{Assessment of bone mineral density}

Considering that free testosterone reflects more accurately the clinical situation than total testosterone in plasma, ${ }^{15}$ the 17 male patients diagnosed as hypogonadal through calculated FT values were considered for assessment of bone mineral density. Three patients were excluded (one patient was excluded on the basis that the primary indication for IDD use was spinal osteoporosis, one patient had the intrathecal opioid therapy discontinued and one patient passed away.

The median age of the 14 patients at the time of BMD assessment was 62.5 years (48-70). All the patients investigated for BMD were Caucasian. The BMI score was $29.4 \mathrm{~kg} / \mathrm{m}^{2}$ (20.1-45.4). According to the BMI score, the majority of patients $(64.3 \%)$ were either overweight or obese and none of the patients were underweight.

Table 2 shows the results of the BMD assessment. Individual $\mathrm{T}$ scores below $-1.0 \mathrm{SD}$ in at least one site were identified in $10(71.4 \%)$ patients. Osteopaenia, defined as a $\mathrm{T}$ score between -1.0 and $-2.5 \mathrm{SD}$, was observed in seven $(50 \%)$ patients. Osteoporosis, defined as a $\mathrm{T}$ score at or below $-2.5 \mathrm{SD}$, was detected in three (21.4\%) patients. When considering the $\mathrm{Z}$ scores, one (7.1\%) of the participants presented a value at or below $-2.5 \mathrm{SD}$, indicating osteoporosis, and four (28.6\%) other patients had Z scores between -1.0 and $-2.5 \mathrm{SD}$ representative of osteopaenia.

Seven patients had $\mathrm{T}$ scores below $-1.0 \mathrm{SD}$ in more than one assessed site (table 3). Three patients had osteoporosis and/or osteopaenia in two sites compared with four patients in three sites. Three of the patients presented $\mathrm{Z}$ scores lower than -1.0 SD in three sites compared with one patient in two sites. No statistical differences were observed between the patients within normal reference range values and those with osteopaenia or osteoporosis for age $(\mathrm{p}=0.72)$ or BMI $(\mathrm{p}=0.48)$.

Several known clinical risk factors for low bone mineral density were present in this sample, including 
Table 2 Bone mineral density measurements

\begin{tabular}{lllr}
\hline Site of measurement & BMD $\left(\mathbf{g} / \mathbf{c m}^{2}\right)$ & T score & Z score \\
\hline Femoral neck $(n=14)$ & $0.925(0.734-1.176)$ & $-1.10(-2.6-0.8)$ & $-0.10(-1.9-2.0)$ \\
Total hip $(n=14)$ & $0.947(0.686-1.222)$ & $-1.10(-3.1-1.0)$ & $-0.40(-2.6-1.9)$ \\
Forearm $(n=10)$ & $0.736(0.665-0.845)$ & $-0.40(-3.2-1.2)$ & $0.30(-2.4-1.7)$ \\
Lumbar $(n=4)$ & $1.185(0.876-1.487)$ & $-0.40(-2.4-2.0)$ & $0.00(-1.9-2.3)$ \\
\hline Statistics are presented as median (minimum-maximum). & & \\
BMD, bone mineral density. & &
\end{tabular}

hypogonadism in all the patients. Investigation of osteoporosis-related fractures through x-rays was not performed. Although the patients in the studied group did not report any incident fractures, assessment of the 10-year probability of major osteoporotic or hip fracture based on the FRAX tool indicated a median probability of $5.7 \%(2.3-17)$ for major osteoporotic fracture and $1.1 \%(0.1-11)$ for hip fracture. Five $(35.7 \%)$ patients were at or above the intervention threshold for hip fracture.

\section{DISCUSSION}

Our study showed that $85 \%$ of male patients on intrathecal opioid therapy were biochemically hypogonadal. The serum gonadotropin ( $\mathrm{LH}$ and FSH) levels in these patients were inappropriately low or low-normal despite low serum testosterone concentrations, suggesting that testosterone suppression was caused through an inhibition of pituitary FSH and LH secretion (secondary hypogonadism). Raised serum prolactin may have contributed to the low testosterone in two patients. Although acute administration of morphine leads to an increase in PRL levels, ${ }^{12}$ tolerance usually develops during chronic administration. ${ }^{20}$ Previous studies investigating the chronic administration of intrathecal morphine have also reported a small proportion of patients with elevated PRL levels. ${ }^{17}{ }^{21}$ In a group of cancer survivors on opioids, $90 \%$ exhibited hypogonadism and low testosterone levels; LH levels, but not FSH levels, were found to be significantly lower when compared with cancer survivors not on opioid therapy. ${ }^{22}$ The important role of endogenous opioids in the control of LH secretion has been demonstrated ${ }^{23}$ and suppression of the hypothalamic-pituitary-gonadal axis by intrathecal opioids may be caused by a mechanism similar to endogenous opioids. ${ }^{24}$ Nevertheless, the suppression of LH levels may be less accentuated when the opioids are administered orally or transdermally rather than intrathecally. $^{12}$

Hypogonadism is an important risk factor for the development of osteoporosis in both sexes. ${ }^{16}$ To our knowledge, the incidence of osteopaenia or osteoporosis in patients undertaking intrathecal opioid therapy has not been reported previously. In our study, $50 \%$ of patients had osteopaenia and $21.4 \%$ had osteoporosis. Interestingly, an association between oral opioid administration and reduced BMD was demonstrated in one study but the presence or absence of hypogonadism was not assessed. ${ }^{25}$ In a cross-sectional study, osteopaenia was present in $50 \%$ of the male patients undertaking oral opioids, ${ }^{26}$ but again it was not clear if those patients were hypogonadal.

An association between oral opioid medication and an increase in fracture risk has also been reported ${ }^{27}$ but assessment of bone mineral density was not performed. The authors suggested that this increase in fracture risk was possibly related to the risk of falls owing to the central nervous system side effect of dizziness caused by oral opioids. Opioid-induced dizziness is less likely to occur in IDD patients since only a fraction of the opioid delivered via the intrathecal route reaches the brain. Low bone mass is an important component of the risk of fracture as well as non-skeletal factors such as the propensity to fall. ${ }^{16}$ Many fragility fractures occur in the absence of osteoporosis, although in the presence of this disease, the risk of fracture is higher. ${ }^{29}$ Osteoporotic fractures are a significant cause of morbidity and mortality, especially in the developed countries, ${ }^{30}$ and are associated with increased mortality, particularly in men. ${ }^{31}$

Table 3 Bone mineral density outcomes

\begin{tabular}{llll}
\hline Site of measurement & Normal & Osteopaenia* & Osteoporosis† \\
\hline Femoral neck $(n=14)$ & $7 / 14(50 \%)$ & $5 / 14(35.7 \%)$ & $2 / 14(14.3 \%)$ \\
Total hip $(n=14)$ & $7 / 14(50 \%)$ & $4 / 14(28.6 \%)$ & $3 / 14(21.4 \%)$ \\
Forearm $(n=10)$ & $7 / 10(70 \%)$ & $2 / 10(20 \%)$ & $1 / 10(10 \%)$ \\
Lumbar $(n=4)$ & $2 / 4(50 \%)$ & $2 / 4(50 \%)$ & \\
\hline
\end{tabular}

Values represent the number of patients/total patients (\%).

${ }^{*}$ Osteopaenia was defined as $-2.5 \mathrm{SD}<\mathrm{T}$ score $<-1.0 \mathrm{SD}$.

†Osteoporosis was defined as T score $\leq-2.5 \mathrm{SD}$. 
The assumption that hypogonadism is a risk factor for decreased BMD has not always been confirmed in the literature. No association between age-related hypogonadism (based on total testosterone) and decreased BMD was found in elderly men. ${ }^{32}$ In contrast, free testosterone (calculated according to the Vermeulen equation) was demonstrated to be an independent predictor of BMD and fractures in elderly men ${ }^{33}$ and a positive predictor of cortical bone size in young men at the age of peak bone mass. ${ }^{34}$ These contradictory findings may have occurred because free testosterone is more important physiologically than total testosterone. SHBG levels, which generally are genetically determined, seem to play an important role in bone mass, hence the reason for free testosterone to be a stronger predictor than total testosterone alone. Recently, it has been suggested that the SHBG levels in healthy adult men at the age of peak bone mass were positively associated with cortical bone size independent of the sex-steroid levels. ${ }^{35}$ However, in middle-aged and elderly men, SHBG elevation was significantly associated with the occurrence of osteoporotic fractures. ${ }^{36}$ Although not yet confirmed, it has been suggested that the effect of SHBG on BMD may change with age and/or testosterone sufficiency or deficiency. ${ }^{37}$

It is important to note the limitations of this study. A small number of patients were included without a control group. The gonadal status and bone mineral density were not evaluated prior to the start of IDD therapy. Information on systemic opioids was not collected. A proportion of these patients are provided with oral opioid medication on an individual basis for occasional flare-ups. The strongest systemic opioid provided is tramadol at a dose $\leq 400 \mathrm{mg} /$ day. Several possible factors may affect the sexual function in this group of patients. Chronic pain did not seem to be the cause of gonadal function reduction in patients undertaking intrathecal morphine therapy when compared with a control group of chronic pain patients who were not taking any form of opioid drugs. ${ }^{24}$ Of the possible chronic illnesses identified in a longitudinal study with 890 male participants, only cancer $(9 \%)$ was associated with a greater decrease in testosterone levels than the decrease that occurred with ageing alone. ${ }^{38}$ Women were not included in this study. Low libido and amenorrhoea have been reported in female IDDS patients, ${ }^{17} 24$ although the prevalence has been reported to be lower in women. ${ }^{26} \mathrm{~A}$ large meta-analysis, which included approximately 39000 men and women, has concluded that the age-specific risk of hip fracture is similar in both men and women with the same BMD and age. ${ }^{39}$ Despite these limitations, the results of BMD assessment suggest that the IDD population may have an increased risk for osteoporotic fractures.

It is important to provide appropriate treatment to patients with low BMD. FRAX analysis is a simple tool that can be used to identify patients in whom osteoporosis prophylaxis is appropriate by taking into account multiple risk factors including BMD levels and hypogonadism.
BMD can be normalised and maintained within the normal range in men with either primary or secondary hypogonadism by continuous, long-term hormonal replacement therapy, ${ }^{40}$ though the full effect on BMD may take up to 24 months. ${ }^{41}$ Opioid-induced hypogonadism may be reversible. Clinically significant improvements in hypogonadal symptoms were observed in men with opioid-induced androgen deficiency following treatment with transdermal testosterone patches. ${ }^{42}$ In patients undertaking intrathecal opioid delivery, recovery of serum testosterone levels following cessation of therapy or significant improvements in libido following hormonal replacement therapy have also been reported. ${ }^{17} 24$

Further studies in this patient group are warranted. Future studies should prospectively evaluate the gonadal axis, as well as the reported sexual health of the participants and BMD. It would also be important to compare these results with matched cohorts of chronic pain patients. Potential comparisons include patients on systemic opioids only, on a different course of intrathecal therapy (eg, ziconotide) or patients using spinal cord stimulation for the management of their chronic pain.

This study suggests an important association between hypogonadism and low bone mass density in patients undertaking intrathecal opioid delivery for the management of chronic non-malignant pain. However, since the gonadal status and BMD were not determined prior to initiation of intrathecal opioid delivery, we cannot conclude that the decreased BMD was caused by hypogonadism or opioid administration. Early detection of hypogonadism followed by appropriate treatment may be of paramount importance to reduce the risk of osteoporosis development and prevention of fractures in this population. Furthermore, surveillance of BMD levels in hypogonadal intrathecal opioid delivery patients should be considered.

Acknowledgements The authors are grateful to $\mathrm{Dr}$ Jane Dale from the Department of Endocrinology and Diabetes at Russells Hall Hospital for her comments on the final version of this manuscript.

Contributors RVD, JHR, JLS, MHL, AJW and RLA have made substantial contributions to (1) the conception and design of the study, or acquisition of data, or analysis and interpretation of data; (2) drafting of the article or revising it critically for important intellectual content and (3) final approval of the version to be submitted.

Funding This research received no specific grant from any funding agency in the public, commercial or not-for-profit sectors.

Competing interests None.

Ethics approval All assessments were performed as part of routine clinical care. No additional procedures were carried out for research purposes.

Provenance and peer review Not commissioned; externally peer reviewed.

Data sharing statement No additional data are available. Data deposited in the Dryad repository: doi:10.5061/dryad.vj7k2.

\section{REFERENCES}

1. Onofrio BM, Yaksh TL, Arnold PG. Continuous low-dose intrathecal morphine administration in the treatment of chronic pain of malignant origin. Mayo Clin Proc 1981;56:516-20. 
2. Grady K, Raphael J. Spinal administration. In: Rice A, ed. Textbook of clinical pain management: chronic volume. London: Hodder Arnold, 2008:284-91.

3. Nordberg G, Hedner T, Mellstrand T, et al. Pharmacokinetic aspects of intrathecal morphine analgesia. Anesthesiology 1984;60:448-54.

4. Bernards C. Cerebrospinal fluid and spinal cord distribution of baclofen and bupivacaine during slow intrathecal infusion in pigs. Anesthesiology 2006;105:169-78.

5. Duarte RV, Raphael JH, Sparkes E, et al. Long-term intrathecal drug administration for chronic nonmalignant pain. J Neurosurg Anesthesiol 2012;24:63-70.

6. Duarte RV, Raphael JH, Haque MS, et al. A predictive model for intrathecal opioid dose escalation for chronic non-cancer pain. Pain Physician 2012;15:363-9.

7. Doleys D, Dinoff B, Page L, et al. Sexual dysfunction and other side effects of intraspinal opiate use in the management of chronic non-cancer pain. Am J Pain Manag 1998;8:5-11.

8. Reddy RG, Aung T, Karavitaki N, et al. Opioid induced hypogonadism. BMJ 2010;341:c4462.

9. Duarte RV, Raphael JH, Mourad L, et al. Prevalence and influence of diagnostic criteria in the assessment of hypogonadism in intrathecal opioid therapy patients. Pain Physician 2013;16:9-14.

10. Katz N, Mazer NA. The impact of opioids on the endocrine system. Clin J Pain 2009;25:170-5.

11. Petak SM, Nankin HR, Spark RF, et al. American Association of Clinical Endocrinologists Medical Guidelines for clinical practice for the evaluation and treatment of hypogonadism in adult male patients2002 update. Endocr Pract 2002:8:440-56.

12. Vuong C, Van Uum SHM, O'Dell LE, et al. The effects of opioids and opioid analogs on animal and human endocrine systems. Endocr Rev 2010;31:98-132.

13. Schulz R, Wilhelm A, Pirke KM, et al. Beta-endorphin and dynorphin control serum luteinizing hormone level in immature female rats. Nature 1981;294:757-9.

14. Pfeiffer DG, Pfeiffer A, Shimohigashi $Y$, et al. Predominant involvement of mu-rather than delta- or kappa-opiate receptors in $\mathrm{LH}$ secretion. Peptides 1983;4:647-9.

15. Vermeulen A, Verdonck L, Kaufman JM. A critical evaluation of simple methods for the estimation of free testosterone in serum. $J$ Clin Endocrinol Metab 1999;84:3666-72.

16. Kanis JA. Diagnosis of osteoporosis and assessment of fracture risk. Lancet 2002;359:1929-36.

17. Abs R, Verhelst $\mathrm{J}$, Maeyaert $\mathrm{J}$, et al. Endocrine consequences of long-term intrathecal administration of opioids. J Clin Endocrinol Metab 2000;85:2215-22.

18. Hackett G, Cole NS, Deshpande AA, et al. Biochemical hypogonadism in men with type 2 diabetes in primary care practice. Br J Diabetes Vasc Dis 2009;9:226-31.

19. Kanis JA, Johnell O, Oden A, et al. FRAX and the assessment of fracture probability in men and women from the UK. Osteoporos Int 2008;19:385-97.

20. Morley JE. The endocrinology of the opiates and opioid peptides. Metabolism 1981;30:195-209.

21. Paice JA, Penn RD, Ryan WG. Altered sexual function and decreased testosterone in patients receiving intraspinal opioids. $J$ Pain Symptom Manage 1994;9:126-31.

22. Rajagopal A, Vassilopoulou-Sellin R, Palmer JL, et al. Symptomatic hypogonadism in male survivors of cancer with chronic exposure to opioids. Cancer 2004;100:851-8.
23. Genazzani AR, Genazzani AD, Volpogni C, et al. Opioid control of gonadotrophin secretion in humans. Hum Reprod 1993;8(Supp 2):151-3.

24. Finch $\mathrm{P}$, Roberts $\mathrm{L}$, Price $\mathrm{L}$, et al. Hypogonadism in patients treated with intrathecal morphine. Clin J Pain 2000;16:251-4.

25. Kinjo M, Setoguchi S, Schneeweiss $\mathrm{S}$, et al. Bone mineral density in subjects using central nervous system-active medications. Am J Med 2005;118:1414.

26. Fraser LA, Morrison D, Morley-Forster $\mathrm{P}$, et al. Oral opioids for chronic non-cancer pain: higher prevalence of hypogonadism in men than in women. Exp Clin Endocrinol Diabetes 2009;117:38-43.

27. Vestergaard P, Rejnmark L, Mosekilde L. Fracture risk associated with the use of morphine and opiates. J Inter Med 2006; 260:76-87.

28. Center JR, Nguyen TV, Sambrook PN, et al. Hormonal and biochemical parameters and osteoporotic fractures in elderly men. $J$ Bone Miner Res 2000;15:5-1411.

29. Kanis JA, Melton LJ, Christiansen C, et al. The diagnosis of osteoporosis. J Bone Miner Res 1994;9:1137-41.

30. Johnell O, Kanis JA. An estimate of the worldwide prevalence and disability associated with osteoporotic fractures. Osteoporos Int 2006;17:1726-33.

31. Center JR, Nguyen TV, Schneider D, et al. Mortality after all major types of osteoporotic fracture in men and women: an observational study. Lancet 1999;353:878-82.

32. Amin S, Zhang Y, Sawin CT, et al. Association of hypogonadism and estradiol levels with bone mineral density in elderly men from the Framingham study. Ann Intern Med 2000;133:951-63.

33. Mellström D, Johnell O, Ljunggren $\mathrm{O}$, et al. Free testosterone is an independent predictor of BMD and prevalent fractures in elderly men: MrOS Sweden. J Bone Miner Res 2006;21:529-35.

34. Lorentzon M, Swanson C, Andersson N, et al. Free testosterone is a positive, whereas free estradiol is a negative, predictor of cortical bone size in young Swedish men: the GOOD study. J Bone Miner Res 2005;20:1334-41.

35. Vanbillemont G, Lapauw B, Bogaert V, et al. Sex hormone-binding globulin as an independent determinant of cortical bone status in men at the age of peak bone mass. J Clin Endocrinol Metab 2010;95:1579-86.

36. Hoppé E, Bouvard B, Royer M, et al. Sex hormone-binding globulin in osteoporosis. Joint Bone Spine 2010;77:306-12.

37. Khosla S. Sex hormone binding globulin: inhibitor or facilitator (or both) of sex steroid action? J Clin Endocrinol Metab 2006;91:4764-6.

38. Harman S, Metter E, Tobin J, et al. Longitudinal effects of aging on serum total and free testosterone levels in healthy men. $J$ Clin Endocrinol Metab 2001;86:724-31.

39. Johnell O, Kanis JA, Oden A, et al. Predictive value of BMD for hip and other fractures. J Bone Miner Res 2005;20:1185-94.

40. Katznelson L, Finkelstein JS, Schoenfeld DA, et al. Increase in bone density and lean body mass during testosterone administration in men with acquired hypogonadism. J Clin Endocrinol Metab 1996;81:4358-65.

41. Snyder PJ, Peachey H, Berlin JA, et al. Effects of testosterone replacement in hypogonadal men. J Clin Endocrinol Metab 2000;85:2670-7.

42. Daniell HW, Lentz R, Mazer NA. Open-label pilot study of testosterone patch therapy in men with opioid-induced androgen deficiency. J Pain 2006;7:200-10. 